\title{
African American gamblers: are they willing to participate in health research?
}

\author{
Amy L Elliott*, Nathan D L Smith, Catherine W Striley, Mirsada Serdarevic and Linda B Cottler \\ Department of Epidemiology, College of Public Health and Health Professions, College of Medicine, University of Florida, USA
}

\begin{abstract}
Despite its severe consequences, gambling disorder is under-researched and under-funded especially among African Americans (AA). We had the opportunity to evaluate gambling among a community sample of AA gamblers and to assess whether gambling impacts their interest in, willingness to participate in, navigation to, and enrollment in health studies. AA recruited into the HealthStreet community engagement program between 2014 and 2017 ( $n=2,661)$ were asked by Community Health Workers about their gambling behaviors and for which types of research studies they would volunteer. Chi-square tests and t-tests were calculated by gambling status for demographics, perceptions of research, navigation to and enrollment in health research. About $34 \%$ of the sample ( $\mathrm{n}=911)$ endorsed lifetime gambling. Compared to AA non-gamblers, AA gamblers were more "definitely interested" in participating in research, more willing to volunteer for three types of studies, and did not require additional remuneration to be enrolled in a study. Though AA gamblers are more willing to participate, they are less likely to be enrolled into health research than AA non-gamblers. This suggests that AA gamblers face increased barriers to enrollment and that researchers may not be using the right avenues to approach and enroll AA gamblers in health research.
\end{abstract}

\section{Introduction}

Gambling disorder affects about one percent of Americans in their lifetime [1]. Another two to three percent of Americans (sometimes called problem gamblers) are affected by symptoms of gambling disorder, although they never reach threshold for disorder [2]. Gambling disorder and problem gambling are associated with several negative outcomes, including: substantial financial losses, bankruptcy, unemployment, comorbid mental health and substance use disorders, broken relationships, increased criminal behavior, and suicide [3-11].

Despite the associated and severe health, social, and economic consequences, gambling disorder is an under-researched and underfunded public health concern [12], especially among racial and ethnic minorities including African Americans [13]. The few studies that exist have revealed an unusual pattern of gambling among African Americans compared to Caucasian Americans, a lower prevalence of gambling, but higher likelihood of meeting criteria for gambling disorder $[8,14]$. In one national study, the conditional prevalence of gambling disorder among individuals who have gambled more than 5 times in any 1 year was more than twice as high in African Americans compared to Caucasian Americans (9\% to 4\%) [8].

Although national studies are important for their generalizability, they often struggle to recruit sufficient numbers of African Americans, who make up approximately $12 \%$ of the US population [15]. Overall, response rates are lower among minority groups and have been declining over time $[15,16]$. This is particularly problematic because gambling disorder has a low base rate in the general population. To illustrate, a representative national study of 10,000 individuals would produce a sample where $0.5-2.5 \%$ had a gambling disorder (approximating a range of national rates). This study with $12 \%$ African Americans (approximating the US population) would produce a sample that included 1200 African Americans, with 6 to 30 having a gambling disorder. Drawing conclusions about gambling disorder in
African Americans based on such a small sample would be problematic and any bias in the sample that led to fewer African Americans or people who met criteria for a gambling disorder would reduce these numbers even further.

Because gambling patterns differ significantly by race and ethnicity, we cannot generalize findings of Caucasians to African Americans. In addition, because African Americans experience gambling-related harms at higher rates than other groups, they bear a disproportionate share of the negative outcomes of gambling $[8,14]$. Thus, gambling disorder deserves further study in this population.

Anecdotal evidence suggests some researchers believe that community recruitment will not lead to high enough rates of enrollment or retention compared to methods like recruitment from treatment centers or undergraduate classes. However, community recruitment of gamblers has been shown to be effective and generalizable to the entire spectrum of gamblers [17-20]. In spite of this, community recruitment of African American gamblers has not been fully realized.

We had the opportunity to evaluate gambling among a community sample of African American gamblers and to assess whether gambling impacts their interest in, willingness to participate in, navigation to, and enrollment in health studies.

${ }^{\star}$ Correspondence to: Amy L. Elliott, Department of Epidemiology, College of Public Health and Health Professions, College of Medicine, University of Florida, 2004 Mowry Road, PO Box 100231, Gainesville, FL 32610, Tel: (352) 273-5468; E-mail: amy.elliott@ufl.edu

Key words: community recruitment, gambling, African Americans, enrollment, research perceptions

Received: October 08, 2018; Accepted: October 18, 2018; Published: October 22,2018 


\section{Materials and Methods}

This analysis was conducted using data from HealthStreet, a community engagement initiative at the University of Florida (UF) that connects community members to relevant medical and social services and health research studies at UF [21-23]. Community Health Workers (CHWs) from HealthStreet meet with community members in frequented public areas like bus stops, shopping plazas, libraries, and parks to describe the purpose of HealthStreet and engage people in conversation. CHWs gather consent and assess health conditions and concerns using the Health Intake Form.

Although one of HealthStreet's main goals is to diversify research by including minority and traditionally underrepresented groups, no one is excluded from participation in HealthStreet. For these analyses, only African Americans at least 18 years old were included. Because questions of the Health Intake Form related to gambling were added in July, 2014, only participants recruited after July were included.

\section{Assessment Tool}

The Health Intake Form was used to assess demographics, top health and neighborhood concerns, health conditions, drug use, past research experience, interest and willingness to participate in research, and gambling history.

\section{Gambling Status}

Gambling status was determined by participant answers to, "Have you more than five times gambled, bet, bought a lottery ticket or used slot machines?" Those who answered yes were categorized as gamblers $(\mathrm{G})$ and those who said no were categorized as non-gamblers (NG). This question format and the more than five times threshold was originally developed for the Epidemiology Catchment Area Study (ECA) in 1979 and has been used with similar constructs like lifetime drug use to indicate a behavior that is "more than experimental."

\section{Health Research Interests and Willingness}

Health research perceptions were also determined by questions on the Health Intake Form. Participants were asked if they had "ever been in a health research study" and responses were grouped as "Yes" or "No/Not Sure." Participants were also asked "how interested are you in being in a research study?" Possible responses were "Definitely," "Maybe," or "Not at all." Only 4\% of the sample answered "Not at all," so responses were grouped as "Definitely" or "Maybe/Not at all" for this analysis. Participants were then asked to respond with a "Yes" or "No" about their willingness to volunteer for eight different types of health research studies, including: ones that required asking questions about health, participating in research for no pay, giving doctors access to medical records, giving a blood sample, giving a sample for genetic studies, taking medicine, staying overnight in a hospital or clinic, and using medical equipment.

Finally, participants were asked what they thought a fair amount of payment was for "participation in a study that lasts about an hour and a half and involves an interview and a blood test." Responses were capped at $\$ 1,000$ for data recording purposes.

\section{Navigation and Enrollment}

Using data collected with the Health Intake Form, the HealthStreet Study Navigator determined if any UF studies match with the participant's health conditions and concerns. The Study Navigator then contacted participants to discuss all aspects of the study, go through initial exclusion criteria, and determine whether participants were interested in the study. If the participant is interested, the Study Navigator forwarded the name and contact information of the participant to the specific UF study coordinator. This process of being linked to a study coordinator is referred to as "navigation." Study coordinators then contacted the participant, went through more detailed exclusion criteria, and described the study more thoroughly.

Enrollment status was sent back to the Study Navigator and recorded in the HealthStreet database. Both study navigation and enrollment status were collected for the current analysis.

\section{Other Covariates}

Lifetime problem gambling status and current gambling status were assessed to help characterize the lifetime gambler group. Lifetime gamblers were asked, "Have you ever had a problem with gambling?" and "Have you gambled in the last 30 days?" Those who said yes to having a problem with gambling were categorized as self-identified lifetime problem gamblers. Those who had gambled in the past 30 days were considered current gamblers.

Age, sex (female or male), marital status (married, widowed/ separated/divorced, or never married), highest level of education (> than 12 years or $\leq 12$ years), any employment in the past 12 months (yes or no), medical insurance status (insured or uninsured), selfperceived health (excellent/good or fair/poor), cigarette smoking in the past 30 days (yes or no), at risk drinking in the past 30 days (men having $>4$ drinks in a day and women having $>3$ drinks in a day), prescription opioid use in the past 30 days (yes or no), and emergency department (ED) visits in the last six months $(<2$ visits or $\geq 2$ visits $)$ were also captured from the Health Intake Form.

\section{Statistical Methods}

Analyses were conducted in SAS version 9.4 (SAS Institute Inc., Cary, NC, USA). Chi-square tests (for categorical variables) and t-tests or median two-sample tests (for continuous variables) were calculated by gambling status for demographics and perceptions of research. Chi-square tests were also calculated by gambling status for study navigation and enrollment.

\section{Results}

\section{Sample Demographics}

A total of 2,661 community members were consented into HealthStreet between July 2014 and February 2017. About 34\% reported gambling more than five times in their lifetime (gamblers; G; $\mathrm{n}=911$ ). The remaining 1,750 participants ( $66 \%$ of the total sample) did not report lifetime gambling (non-gamblers; NG).

Table 1 shows demographic characteristics overall and by selfreported gambling status. The average age of the sample was 46.4 years old, ranging from 18 to 91 years of age. A large proportion of participants were never married $(48.5 \%)$. Over half were female (64.1\%), unemployed (64.8\%), insured (64.6\%), had 12 or less years of education $(64.4 \%)$, or perceived their health to be good or excellent (59.3\%). Close to a fourth engaged in cigarette smoking $(29.6 \%)$ or at risk drinking behavior (23.1\%) in the past 30 days. About $15 \%$ had used a prescription opioid in the past 30 days or had been seen in the ED (emergency department) at least twice in the past six months. About 
Table 1. Sample demographics by gambling status among African American community members

\begin{tabular}{|c|c|c|c|c|}
\hline & Total N=2661 (100.0) & $\begin{array}{c}\text { Non-Gamblers } \\
n=1750(65.8)\end{array}$ & $\begin{array}{c}\text { Gamblers } \\
\mathrm{n}=911(34.2)\end{array}$ & Chisq or T-test \\
\hline & $\mathrm{n}(\%)$ & $\mathrm{n}(\%)$ & $\mathrm{n}(\%)$ & $\mathrm{p}$ value \\
\hline Age mean $( \pm$ SD) [range] & $46.4(15.2)[18-91]$ & $45.3(15.8)[18-91]$ & $48.6(13.8)[18-84]$ & $<0.0001$ \\
\hline Female & $1707(64.1)$ & $1192(68.1)$ & $515(56.4)$ & $<0.0001$ \\
\hline $\begin{array}{l}\text { Marital Status: } \\
\text { Married } \\
\text { Widowed/Separated/Divorced } \\
\text { Never Married }\end{array}$ & $\begin{array}{c}515(18.4) \\
853(32.1) \\
1288(48.5)\end{array}$ & $\begin{array}{l}328(18.8) \\
530(30.3) \\
890(50.9)\end{array}$ & $\begin{array}{l}187(20.6) \\
323(35.6) \\
398(43.8)\end{array}$ & 0.0021 \\
\hline $\begin{array}{l}\text { Highest Level of Education: } \\
>12 \text { years } \\
\leq 12 \text { years }\end{array}$ & $\begin{array}{c}950(35.6) \\
1718(64.4)\end{array}$ & $\begin{array}{c}611(34.8) \\
1144(65.2)\end{array}$ & $\begin{array}{l}339(37.1) \\
574(62.9)\end{array}$ & 0.2360 \\
\hline Any Employment in Past 12 Months & $936(35.2)$ & $606(34.6)$ & $330(36.2)$ & 0.4076 \\
\hline Any Medical Insurance & $1722(64.6)$ & $1123(64.1)$ & $599(65.6)$ & 0.4504 \\
\hline $\begin{array}{l}\text { Self - Perceived Health: } \\
\text { Excellent/Good } \\
\text { Fair/Poor }\end{array}$ & $\begin{array}{l}1571(59.3) \\
1077(40.7)\end{array}$ & $\begin{array}{c}1038(59.6) \\
704(40.4)\end{array}$ & $\begin{array}{l}533(58.8) \\
373(40.7)\end{array}$ & 0.7069 \\
\hline Smoked Cigarettes in Past 30 Days & $788(29.6)$ & $462(26.3)$ & $326(35.8)$ & $<0.0001$ \\
\hline At Risk Drinking in Past 30 Days & $616(23.1)$ & $341(19.5)$ & $275(30.1)$ & $<0.0001$ \\
\hline Prescription Opioid Use in the Past 30 Days & $394(14.8)$ & $234(13.4)$ & $160(17.5)$ & 0.0041 \\
\hline Been to the ED at Least Twice in the Past 6 months & $421(15.8)$ & $244(13.9)$ & $177(19.4)$ & 0.0002 \\
\hline Self-Identified Lifetime Problem Gambler & N/A & N/A & $75(8.2)$ & N/A \\
\hline Gambled in the Past 30 Days & N/A & N/A & $370(40.8)$ & N/A \\
\hline
\end{tabular}

$8 \%$ of lifetime gamblers self-identified as lifetime problem gamblers $(\mathrm{n}=75) ; 40.8 \%$ of the gamblers gambled in the past 30 days $(\mathrm{n}=370)$.

Significant differences between gambling groups were found for age, sex, marital status, past six months ED visits, past 30 day prescription opioid use, and smoking and drinking in the past 30 days. When compared to AA non-gamblers, AA gamblers were older; more likely to be male; more likely to endorse being widowed, separated, or divorced; more likely to have smoked or had at risk drinking patterns in the past 30 days; more likely to have used a prescription opioid in the last 30 days; and more likely to have visited the ED at least twice in the past six months (Table 1).

\section{Health Research Perceptions by Problem Gambling Status}

As shown in Table 2, there were few significant differences by gambling status in health research perceptions among AAs. About $18 \%$ of the overall sample had participated in a research study before joining HealthStreet. Gamblers were more likely to be definitely interested in currently participating in a research study than non-gamblers (G: 52.5\%; NG: 45.4\%; $\left.\chi^{2}(1, \mathrm{n}=2661)=12.16\right)$. There were no differences in what groups considered a fair amount for a study that lasted about 1.5 hours and involved an interview and a blood test. Both gamblers and non-gamblers had response distributions skewed to the right with a median response of $\$ 50$ and a range of $\$ 0$ to $\$ 1000$.

Overall, participants were very willing to volunteer for all of the study types, with group willingness ranging from 59.8\% (NG) for a study that requires medication use to $94.8 \%(\mathrm{G})$ for a study that only asks questions about health. There were no significant differences by gambling status in willingness to volunteer for a health study that only asked questions about health $(94.4 \%)$, allowed researchers to access medical records $(87.5 \%)$, required a blood sample $(85.2 \%)$ or a genetic sample $(85.6 \%)$, and even if there was no pay (77.0\%). However, gamblers were more willing than non-gamblers to volunteer for a study that required taking an experimental medication (G: $63.9 \%$; NG: $59.8 \% ; \chi 2(1, \mathrm{n}=2661)=4.23)$, an overnight stay at a hospital or clinic (G: 77.6\%; NG: 71.3\%; $\chi 2(1, \mathrm{n}=2661)=12.21)$, or that used medical equipment (G: 88.6\%; NG: 84.3\%; $\chi 2(1, \mathrm{n}=2661)=8.96)($ Table 2$)$.

As shown in Table 3, 530 gamblers and 739 non-gamblers were navigated to UF studies during the four year study period. While gamblers were more likely to be navigated to a UF study than nongamblers (G: 58.2\%; NG: 42.2\%; $\left.\chi^{2}(1, \mathrm{n}=2661)=61.09\right)$, once navigated, they were less likely to be enrolled in UF studies (G: $35.9 \%$; NG: 49.5\%; $\chi 2(1, \mathrm{n}=1269)=23.45)($ Table 3$)$.

\section{Discussion}

In less than 4 years, over 900 African American lifetime gamblers were recruited from the community by CHWs. HealthStreet's main catchment area offers few high-stakes gambling opportunities and HealthStreet CHWs do not seek out gamblers for enrollment; however, HealthStreet CHWs were successful in recruiting each month over 21 lifetime gamblers (or 8 current gamblers). This analysis shows that researchers can work to enhance the representativeness of gambling studies by recruiting gamblers, and African American gamblers in particular, from the community. Community engagement programs like HealthStreet help engage African American gamblers and assist with their enrollment process.

Although $47.8 \%$ of African American gamblers from the community were "definitely interested" in participating in health research, only $18.1 \%$ had been involved in a health study in the past. When compared to African American non-gamblers in the community, African American gamblers were more "definitely interested" in participating in research, more willing to volunteer for three types of studies, and did not require additional remuneration to be enrolled in a hypothetical study. Despite their increased interest and willingness to participate, gamblers have not been enrolled more in the past than their non-gambling peers, suggesting that researchers are not using the right avenues to approach and enroll them.

In addition, through a community engagement model developed to help enroll populations commonly under-recruited in research, African American gamblers have shown higher intention to enroll in health research studies (shown through higher navigation to UF study coordinators) than their non-gambling peers, yet fewer African 
Table 2. Perceptions of health research by gambling status among African American community members

\begin{tabular}{|c|c|c|c|c|}
\hline & Total N=2661 (100.0) & $\begin{array}{l}\text { Non-Gamblers } \\
\mathrm{n}=1750(65.8)\end{array}$ & $\begin{array}{c}\text { Gamblers } \\
\mathrm{n}=911(34.2)\end{array}$ & $\begin{array}{l}\text { Chisq or median } \\
\text { two-sample test }\end{array}$ \\
\hline & $\mathrm{n}(\%)$ & $\mathrm{n}(\%)$ & $\mathrm{n}(\%)$ & $\mathrm{p}$ value \\
\hline \multicolumn{5}{|l|}{ Past Experience and Current Perceptions } \\
\hline Has ever been in health research study & $475(18.1)$ & $299(17.3)$ & $176(19.6)$ & 0.1329 \\
\hline Definitely interested in participating in research study & $1276(47.8)$ & $797(45.4)$ & $479(52.5)$ & 0.0005 \\
\hline $\begin{array}{l}\text { Fair amount for study that lasts about } 1.5 \text { hours and involves an interview } \\
\text { and a blood test [median (range)] }\end{array}$ & $\$ 50(\$ 0-\$ 1000)$ & $\$ 50(\$ 0-\$ 1000)$ & $\$ 50(\$ 0-\$ 1000)$ & 0.0940 \\
\hline \multicolumn{5}{|l|}{ Would volunteer for a health research study... } \\
\hline That only asked questions about your health & $2514(94.4)$ & $1650(94.2)$ & $864(94.8)$ & 0.5153 \\
\hline If researchers wanted to see your medical records & $2328(87.5)$ & $1524(87.0)$ & $804(88.4)$ & 0.3131 \\
\hline If you had to give a blood sample & $2268(85.2)$ & $1475(84.3)$ & $793(87.1)$ & 0.0546 \\
\hline If you were asked to give a sample for genetic studies & $2280(85.6)$ & $1487(84.9)$ & $793(86.9)$ & 0.1672 \\
\hline If you might have to take medicine & $1626(61.2)$ & $1045(59.8)$ & $581(63.9)$ & 0.0397 \\
\hline If you were asked to stay overnight in a hospital or clinic & $1957(73.5)$ & $1250(71.3)$ & 707 (77.6) & 0.0005 \\
\hline If you might have to use medical equipment & $2282(85.7)$ & $1478(84.3)$ & $804(88.6)$ & 0.0028 \\
\hline If you weren't paid & $2047(77.0)$ & $1362(77.9)$ & $685(75.3)$ & 0.1310 \\
\hline
\end{tabular}

Table 3. Navigation and enrollment by gambling status among African American community members

\begin{tabular}{|c|c|c|c|c|}
\hline & $\begin{array}{c}\text { Total } \\
\text { N=2661 (100.0) n (\%) }\end{array}$ & $\begin{array}{c}\text { Non-Gamblers } \\
\mathrm{n}=1750(65.8) \mathrm{n}(\%)\end{array}$ & $\begin{array}{c}\text { Gamblers } \\
\mathrm{n}=911(34.2) \mathrm{n}(\%)\end{array}$ & Chisq \\
\hline Ever Navigated & $1269(47.7)$ & $739(42.2)$ & $530(58.2)$ & $<0.0001$ \\
\hline Ever Enrolled (Conditional Upon Navigation) & $556(43.8)$ & $366(49.5)$ & $190(35.9)$ & $<0.0001$ \\
\hline
\end{tabular}

American gamblers have been enrolled in research than non-gamblers. This suggests that African American gamblers are excluded from more studies than their non-gambling peers and/or they face increased barriers to enrollment (like lack of transportation, no child care, or poorer health).

\section{Limitations and Strengths}

Findings must be reviewed in the light of a few limitations. First, there may be better ways to assess gambling and problem gambling than by asking if a participant has gambled at least five times in their lifetime and if they have ever had a problem with gambling. A different measure of disordered gambling such as the Canadian Problem Gambling Index [24] or a more succinct screen such as the BriefBiosocial Gambling Screen [25] may reveal slightly different results in research willingness. Second, the HealthStreet Health Intake Form does not specifically ask about willingness to volunteer for a gambling study, so we have assumed that interest and willingness to participate in health studies can be used as a proxy for interest and willingness to participate in gambling studies.

Limitations notwithstanding, this analysis has many strengths including that it has added to the literature in two main ways: it is one of the first papers to discuss feasibility of recruiting African American gamblers from the community and it is the first to examine research perceptions of African Americans stratified by gambling status. It is particularly important to the future of gambling research as it demonstrates that African American gamblers can be recruited from the community, even communities lacking gambling opportunities.

\section{Future Studies}

Future studies should examine the feasibility of recruiting African Americans from the community with gambling disorder or problem gambling. Use of a structured interview with Diagnostic and Statistical Manual of Mental Disorders 5 (DSM 5) criteria for gambling disorder such as the Diagnostic Interview Schedule (DIS+SAM 5) [26] may allow for better comparison between studies. Future studies should also assess African American community members' willingness to volunteer for gambling studies in particular to confirm that interest and willingness to participate in health studies can be used as a proxy for interest and willingness to participate in gambling studies. In addition, future analyses should focus on barriers to enrollment and reasons for exclusion among African Americans by gambling status.

\section{Acknowledgements}

None

\section{Funding}

Funding was provided by the Clinical and Translational Science Institute (CTSI) and the University of Florida (UF) College of Public Health \& Health Professions and College of Medicine. CTSI is supported in part by the NIH/NCATS Clinical and Translational Science Award to the University of Florida grants UL1 TR000064, KL2 TR000065 and TL1 TR000066.

This research was supported by the NIDA T32 training grant at the UF Substance Abuse Training Center in Public Health from the National Institutes of Health (T32DA035167). The content is solely the responsibility of the authors and does not necessarily represent the official view of the NIH.

\section{Disclosure Statement}

The authors declare they have no conflict of interest.

\section{Ethical Approval}

All procedures performed in studies involving human participants were in accordance with the ethical standards of the institutional and/ or national research committee and with the 1964 Helsinki declaration and its later amendments or comparable ethical standards.

\section{References}

1. American Psychiatric Association (2013) Diagnostic and statistical manual of menta disorders (5th Edn) American Psychiatric Publishing, Arlington, VA. 
2. Shaffer HJ, Hall MN, Vander Bilt J (1999) Estimating the prevalence of disordered gambling behavior in the United States and Canada: a research synthesis. American Journal of Public Health 89: 1369-1376.

3. Vaddiparti K, Cottler LB (2017) Personality disorders and pathological gambling. Curr Opin Psychiatry 30: 45-49. [Crossref]

4. Vaddiparti K, Striley CW, Cottler LB (2016) Association between gambling and exposure to guns among cocaine-using women. Violence Gend 1: 162-166.

5. Hodgins DC, Stea JN, Grant JE (2011) Gambling disorders. Lancet 378: 1874-1884. [Crossref]

6. Kessler RC, Hwang I, LaBrie R, Petukhova M, Sampson NA, et al. (2008) DSM-IV pathological gambling in the National Comorbidity Survey Replication. Psychol Med 38: 1351-1360. [Crossref]

7. Petry N, Stinson F, Grant B (2005) Comorbidity of DSM-IV pathological gambling and other psychiatric disorders: results from the national epidemiologic survey on alcohol and related conditions. Journal of Clinical Psychiatry 66: 564-574.

8. Alegria A, Petry N, Hasin D, Liu S, Grant B, et al. (2009) Disordered gambling among racial and ethnic groups in the US: results from the national epidemiologic survey on alcohol and related conditions. CNS Spectrums 14: 132-142.

9. Cunningham-Williams RM, Gattis MN, Dore PM, Shi P, Spitznagel EL Jr (2009) Towards DSM-V: considering other withdrawal-like symptoms of pathological gambling disorder. Int J Methods Psychiatr Res 18: 13-22. [Crossref]

10. Cunningham-Williams RM, Cottler LB (2001) The epidemiology of pathologica gambling. Semin Clin Neuropsychiatry 6: 155-166. [Crossref]

11. Cunningham-Williams RM, Grucza RA, Cottler LB, Womack SB, Books SJ, et al. (2005) Prevalence and predictors of pathological gambling: results from the St. Louis Personality, Health and Lifestyle (SLPHL) Study. J Psychiatr Res 39: 377-390.

12. Petry NM, Blanco C (2013) National gambling experiences in the United States: will history repeat itself? Gambling in the US. Addiction 108: 1032-1037.

13. Cottler LB (2003) Toward an improved understanding of comorbidity. Journal of Gambling Studies 19: 257-260.

14. Welte J, Barnes G, Wieczorek W, Tidwell MC, Parker J (2001) Alcohol and gambling pathology among U.S. adults: prevalence, demographic patterns and comorbidity. $J$ Stud Alcohol 62: 706-712. [Crossref]
15. Graham C (2006) Inpatient survey 2005 additional analysis. Resource document Picker Institute Europe Acute Coordination Centre unpublished report.

16. Fowler FJ (2014) Survey Research Methods. (5th Edn) California, Los Angeles.

17. McGrath DS, Kim HS, Boothby CA, Romanow NK, Hodgins DC (2016) Patterns and motives for electronic cigarette use in a sample of community-recruited gamblers. International Journal of Mental Health and Addiction 6: 276-283.

18. Ledgerwood DM, Milosevic A (2015) Clinical and personality characteristics associated with post-traumatic stress disorder in problem and pathological gamblers recruited from the community. Journal of Gambling Studies 31: 501-512.

19. Gainsbury SM, Russell A, Blaszczynski A (2014) Are psychology university student gamblers representative of non-university students and general gamblers? A comparative analysis. Journal of Gambling Studies 30: 11-25.

20. Goodie AS, MacKillop J, Miller JD, Fortune EE, Maples J, et al. (2013) Evaluating the south oaks gambling screen with DSM-IV and DSM-5 criteria: results from a diverse community sample of gamblers. Assessment 20: 523-531.

21. Cottler LB, Nagarajan R (2012) Real-time assessment of community health needs and concerns. Science Translational Medicine 4: $119 \mathrm{mr}$.

22. Webb FJ, Khubchandani J, Striley CW, Cottler LB (2018) Black-White Differences in Willingness to Participate and Perceptions About Health Research: Results from the Population-Based HealthStreet Study. J Immigr Minor Health.

23. Serdarevic M, Gurka KK, Striley CW, Vaddiparti K, Cottler LB (2018) Prevalence of Concurrent Prescription Opioid and Hazardous Alcohol Use Among Older Women: Results from a Cross-Sectional Study of Community Members. J of Community Health.

24. Ferris JA, Wynne HJ (2001) The Canadian problem gambling index. Canadian Centre on Substance Abuse, Ottawa, ON pp. 1-59.

25. Gebauer L, LaBrie R, Shaffer HJ (2010) Optimizing DSM-IV-TR classification accuracy: a brief biosocial screen for detecting current gambling disorders among gamblers in the general household population. Can J Psychiatry 55: 82-90. [Crossref]

26. Cottler LB, Striley CW, North C (2016) Diagnostic Interview Schedule for the DSM-5 (DIS+SAM 5). University of Florida, Gainesville, FL.

Copyright: $(2018$ Elliott AL. This is an open-access article distributed under the terms of the Creative Commons Attribution License, which permits unrestricted use, distribution, and reproduction in any medium, provided the original author and source are credited. 